\title{
Improved Aqueous Solubility and Antihypercholesterolemic Activity of Ezetimibe on Formulating with Hydroxypropyl- $\beta$-Cyclodextrin and Hydrophilic Auxiliary Substances
}

\author{
Kale Mohana Raghava Srivalli ${ }^{1}$ and Brahmeshwar Mishra ${ }^{1,2}$
}

\begin{abstract}
Received 16 February 2015; accepted 28 May 2015; published online 16 June 2015
Abstract. The purpose of this study was to improve the aqueous solubility, dissolution, and pharmacodynamic properties of a BCS class II drug, ezetimibe (Eze) by preparing ternary cyclodextrin complex systems. We investigated the potential synergistic effect of two novel hydrophilic auxiliary substances, D- $\alpha-$ tocopheryl polyethylene glycol 1000 succinate (TPGS) and L-ascorbic acid-2-glucoside (AA2G) on hydroxypropyl- $\beta$-cyclodextrin (HPBCD) solubilization of poorly water-soluble hypocholesterolemic drug, Eze. In solution state, the binary and ternary systems were analyzed by phase solubility studies and Job's plot. The solid complexes prepared by freeze-drying were characterized by Fourier transform infrared (FTIR), differential scanning calorimetry (DSC), powder X-ray diffraction (XRD), nuclear magnetic resonance (NMR), and scanning electron microscopy (SEM). The log $P$ values, aqueous solubility, dissolution, and antihypercholesterolemic activity of all systems were studied. The analytical techniques confirmed the formation of inclusion complexes in the binary and ternary systems. HPBCD complexation significantly $(p<0.05)$ reduced the $\log P$ and improved the solubility, dissolution, and hypocholesterolemic properties of Eze, and the addition of ternary component produced further significant improvement $(p<0.05)$ even compared to binary system. The remarkable reduction in $\log P$ and enhancement in solubility, dissolution, and antihypercholesterolemic activity due to the addition of TPGS or AA2G may be attributed to enhanced wetting, dispersibility, and complete amorphization. The use of TPGS or AA2G as ternary hydrophilic auxiliary substances improved the HPBCD solubilization and antihypercholesterolemic activity of Eze.
\end{abstract}

KEY WORDS: dissolution; ezetimibe-HPBCD; ezetimibe-HPBCD-AA2G; ezetimibe-HPBCD-TPGS; phase solubility.

\section{INTRODUCTION}

The drug ezetimibe (Eze) chosen in the present study, is a model BCS class II drug with low water solubility. Eze is chemically 1-(4-fluorophenyl)-3(R)-[3-(4-fluorophenyl)-3(S)hydroxypropyl]-4(S)-(4-hydroxyphenyl)-2-azetidinone [1] and the structural formula is shown in Fig. 1. It is the first-ofits-kind hypocholesterolemic that serves as a cholesterol absorption inhibitor unlike other marketed lipid lowering agents that act by inhibiting the synthesis of cholesterol. Eze inhibits cholesterol absorption by the small intestine, but, being a Pglycoprotein (P-gp) substrate, the in vivo absorption of Eze is lowered by P-gp efflux at the small intestinal brush border. The oral bioavailability of Eze is lowered to as low as $35 \%$ due to its low aqueous solubility and P-gp efflux [2].

Cyclodextrin (CD) complexation of non-polar drug molecules has been well-known to render the drugs more soluble by several orders of magnitude when compared to the parent

\footnotetext{
${ }^{1}$ Department of Pharmaceutics, Indian Institute of Technology (Banaras Hindu University), Varanasi, Uttar Pradesh 221005, India.

${ }^{2}$ To whom correspondence should be addressed. (e-mail: bmishrabhu@rediffmail.com)
}

or uncomplexed drug molecules. CDs are highly water-soluble polymers that can improve the solvation of dissolved drug molecules with the ability to stabilize supersaturated solutions and inhibit precipitation [3]. Hydroxypropyl- $\beta$-cyclodextrin (HPBCD) presents the highest aqueous solubility $(>60 \%$ at $25^{\circ} \mathrm{C}$ ) among the natural CDs and their derivatives and has been employed in several marketed pharmaceutical products [4]. Attempts were made in the past to study the benefits of Eze-CD complexation. Patel et al. was the first to prepare incompletely amorphous complexes of Eze with $\beta-C D$ and HPBCD by coevaporation and kneading methods and pointed out the influence of method of complex preparation on solubility and dissolution behaviors [5]. Taupitz et al. also prepared incompletely amorphous Eze-HPBCD binary complex by freeze-drying method and clearly stated that incomplete amorphous state relates to incomplete complexation of the sample [6]. Such incomplete complexation may be in turn related to the choice of solvents used to solubilize Eze as explained by Selic et al. [7]. Methanol or ethanol was used in the aforementioned studies and these solvents were said to be associated with precipitation of either Eze or HPBCD during mixing or lyophilization process [7]. Tertiary butyl alcohol (TBA) was shown as a suitable solvent by Selic et al. where 


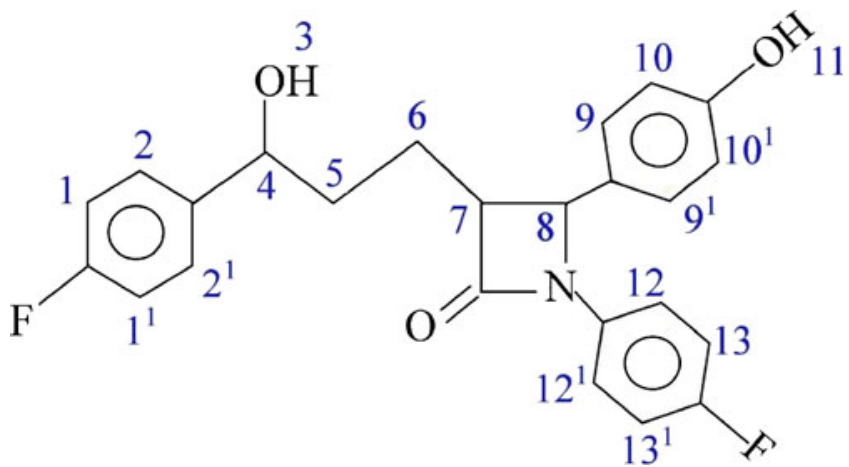

Fig. 1. Structure of ezetimibe showing proton assignments

complete amorphous state of Eze-HPBCD complex was reported [7], and the same was used in our present study and we performed an elaborated analysis of the complexes. Proton nuclear magnetic resonance (H-NMR) spectroscopy, scanning electron microscopy (SEM), saturation solubility, and dissolution studies were not studied by Selic et al. To the best of our knowledge, the antihypercholesterolemic activity of neither binary nor ternary $\mathrm{CD}$ complexes of Eze was reported till date.

Minimizing the amounts of high molecular weight CDs in formulations without compromising on the solubility advantage of CD complexes is of pharmaceutical importance, and it may be possible by introducing auxiliary substances into binary inclusion complexes to form supramolecular ternary systems. The ternary systems may further improve the physicochemical and transport properties of drugs in comparison to binary complexes [8]. Literature reports several studies on the effect of water-soluble substances like polymers [9-11], surfactants [12], metal salts [13], and amino acids [14] on CD solubilization of drugs. In the present study, we evaluated the effect of two hydrophilic auxiliary substances, namely, D- $\alpha$ tocopheryl polyethylene glycol 1000 succinate (Vitamin E TPGS or simply TPGS) and L-ascorbic acid-2-glucoside (AA2G), as third components to the CD complexes, for the first time. Their effect on HPBCD solubilization of Eze was investigated. TPGS is a novel lipid-based, highly water-soluble, non-ionic surfactant that has been approved as safe excipient by US FDA. It also exhibits P-gp inhibitory action and has been widely known to increase the solubility and bioavailability of water-insoluble drugs by many folds $[15,16]$. AA2G is a facile hydrophilic excipient that has been approved as a food additive and is expected to be used as a principal ingredient for solubilization in fat-soluble vitamin formulations and in other cosmetic products [17].

Eze is a hypocholesterolemic P-gp substrate, HPBCD is known to maintain cholesterol homeostasis [18], TPGS is a Pgp inhibitor, and AA2G is an efficient solubilizer. So, we hypothesized that the complexes may serve to not only improve the solubility and release properties of Eze in vitro but also to enhance the pharmacodynamic performance of Eze by offering synergistic hypocholesterolemic effect or improving its in vivo absorption at the small intestinal brush border. The objective of the current study was to prepare binary, Eze-HPBCD (E-CD), and ternary, Eze-HPBCD-TPGS (E-CD-TPGS) and EzeHPBCD-AA2G (E-CD-AA2G), complexes and study their solubility and dissolution properties. Freeze-drying was the method of preparation and the complexes were evaluated for solid-state characteristics and antihypercholesterolemic activity.

\section{MATERIALS AND METHODS}

\section{Materials}

Eze (purity $=99.3 \%$ ) was a kind gift from Lupin Ltd. (Pune, India). HPBCD (DS=5.04), TPGS, and AA2G were received as generous gift samples from Gangwal Chemicals Pvt. Ltd. (Mumbai, India), Antares Health Products, Inc. (IL, USA), and Nagase Pvt. Ltd. (Mumbai, India), respectively. All other materials of analytical reagent grade were purchased locally and used as received.

\section{Methods}

Evaluating the Effect of Increasing Concentration of TPGS or $A A 2 G$ with Fixed Concentration of HPBCD

A previously reported method was adopted with modifications [19]. Excess Eze $(20 \mathrm{mg})$ was added to $10 \mathrm{~mL}$ acetate buffer solutions of $\mathrm{pH} 4.5$, containing a fixed concentration of HPBCD $(2 \% w / v)$ and increasing amounts of TPGS $(0.01 \%-$ $0.25 \% w / v)$ or AA2G $(0.01 \%-0.5 \% w / v)$. TPGS and AA2G were studied at different concentrations owing to their differing molecular weights. The suspensions were shaken on a rotary shaker continuously for 1 week to obtain equilibrium at room temperature $\left(25^{\circ} \mathrm{C} \pm 1{ }^{\circ} \mathrm{C}\right)$. The unsolubilized drug in the suspensions was then filtered with syringe through a nylon membrane filter $(0.45 \mu \mathrm{m})$. The filtrates, after appropriate dilutions, were analyzed by UV at $232 \mathrm{~nm}$.

\section{Phase Solubility Studies}

The method reported by Higuchi and Connors [20] was followed. Excess amount of Eze $(20 \mathrm{mg})$ was added to $10 \mathrm{~mL}$ acetate buffer solutions of $\mathrm{pH} 4.5$, containing 2-14 mM HPBCD (liquid state E-CD system) with or without the addition of $0.05 \% w / v$ TPGS (liquid state E-CD-TPGS system) or $0.1 \% w / v$ AA2G (liquid state E-CD-AA2G system). The suspensions were continuously shaken on a rotary shaker for 1 week at room temperature $\left(25^{\circ} \mathrm{C} \pm 1^{\circ} \mathrm{C}\right)$ to obtain equilibrium. The suspensions were then filtered, appropriately diluted, and analyzed by UV at $232 \mathrm{~nm}$. The experiments were performed in triplicate and the straight line portions of the phase solubility (PS) curves were used to calculate the apparent stability constants $(K)$ of the binary and ternary complexes as per the following equation.

$$
K=\text { slope } / S_{0}(1-\text { slope })
$$

$S_{0}$ is the intrinsic solubility of Eze.

Job's and Benesi-Hildebrand plots were also constructed to confirm the stoichiometric ratio of E-CD in the binary and ternary systems [21-23].

\section{Preparation of Inclusion Complexes in Solid State}

The optimal ratio of Eze and HPBCD in the binary and ternary systems was determined based on the PS studies as well as the UV-visible spectroscopy-based continuous variation method (Job's plot). The complexes were prepared by the 
widely employed freeze-drying method [24, 25]; 1 mmol Eze dissolved in TBA and $2 \mathrm{mmol}$ HPBCD (1 mmol HPBCD for E-CD-AA2G system) in distilled water were mixed at $30^{\circ} \mathrm{C}$ and stirred for $30 \mathrm{~min}$ to ensure a homogenous solution. The concentration of the drug solution was $25 \mathrm{mg} / \mathrm{mL}$ and that of the HPBCD solution was $300 \mathrm{mg} / \mathrm{mL}$. The solution was cooled to room temperature, filtered, prefrozen, and then lyophilized. The preparation of ternary complexes was similar to that of binary complex except that $0.05 \% w / v$ TPGS, in case of ECD-TPGS ternary system, and $0.1 \% w / v$ AA2G, in case of ECD-AA2G system, were added, respectively, to the $300 \mathrm{mg} /$ mL HPBCD solution, before mixing it with the drug solution. The resultant product was a fine, white powder in all the cases.

\section{Characterization}

Fourier transform infrared (FTIR) spectra were recorded using an FTIR spectrophotometer (FTIR-8400S, Shimadzu Co., Kyoto, Japan), over the range of $4000-400 \mathrm{~cm}^{-1}$ by cogrinding with anhydrous $\mathrm{KBr}$ and pelletizing the samples. NMR spectral data was obtained on a $300-\mathrm{MHz}$ NMR spectrometer (Jeol FT-NMR AL-300, Japan) at $25^{\circ} \mathrm{C}$ by dissolving the samples in dimethylsulfoxide and using tetramethylsilane as internal reference. Differential scanning calorimetry (DSC) analysis was performed using DSC-822 ${ }^{\mathrm{e}}$ (Mettler Toledo, AG, Analytical, Switzerland) by heating the samples between $10^{\circ} \mathrm{C}$ and $300^{\circ} \mathrm{C}$. Powder X-ray diffraction (XRD) patterns were traced by employing X-ray diffractometer (PW3050/60 X'pert PRO, PANalytical, Netherlands) with $\mathrm{Cu}$ anode at $40 \mathrm{kV}$ and $30 \mathrm{~mA}$. The surface morphological properties were investigated by SEM (FEI, QUANTA-200, Netherlands).

\section{Drug Content}

Known amounts of binary and ternary systems equivalent to $10 \mathrm{mg}$ of drug were dissolved in $5 \mathrm{~mL}$ methanol, sonicated for $1 \mathrm{~min}$, and filtered. After appropriate dilutions, the solutions were assayed for Eze content by UV at $232 \mathrm{~nm}$. The readings were taken in triplicate and the average was noted.

\section{Measurement of Octanol-Water Partition Coefficient $(P)$ and $\log P$}

Five-milliliter aqueous solutions of each system (pure Eze, binary and ternary complexes), at $10^{-4} \mathrm{~mol} / \mathrm{L}$ concentration, were, respectively, mixed with $5 \mathrm{~mL}$ octanol at room temperature and shaken vigorously in a separating funnel to reach equilibrium. The systems were allowed to stand under gravity to separate the two phases, and the amount of drug in each phase was quantified by UV at $232 \mathrm{~nm}$. $\log P$, the logarithm of partition coefficient $(P)$, was calculated using the following equation:

$\log P=\log$ (Drug concentration in octanol phase/Drug concentration in aqueous phase )

\section{Saturation Solubility Studies}

Excess amounts of drug and binary and ternary systems were added to $10 \mathrm{~mL}$ distilled water. The suspensions were shaken continuously on a rotary shaker for 1 week at room temperature and filtered, and the drug amount was measured by UV at $232 \mathrm{~nm}$.

\section{Dissolution}

The method of dissolution reported for Eze in the "Dissolution Methods" guide of FDA was adapted with modifications [26]. The dissolution studies were carried out by filling pure Eze or each of the formulations equivalent to $10 \mathrm{mg}$ of Eze into hard gelatin capsules. Five hundred milliliters of acetate buffer of $\mathrm{pH} 4.5$, containing $0.45 \% \mathrm{w} / v$ sodium lauryl sulfate (SLS), was used as the medium, and dissolution was conducted using the USP apparatus I (Electrolab TDT$08 \mathrm{~L}$, India) at $37^{\circ} \mathrm{C} \pm 0.5^{\circ} \mathrm{C}$ and $100 \mathrm{rpm}$ rotation rate. Fivemilliliter samples were withdrawn at appropriate time intervals and the dissolution medium volume was made up to $500 \mathrm{~mL}$ by replacing the withdrawn samples with fresh medium. The collected samples were filtered and appropriately diluted, and Eze content was quantified by UV at $232 \mathrm{~nm}$. The percent drug dissolved at each time point was calculated for all the formulations and the data obtained by six replicate determinations was averaged and recorded.

\section{Antihypercholesterolemic Activity}

The study protocol was approved and guided by the Central Animal Ethical Committee, Institute of Medical Sciences, Banaras Hindu University, Varanasi, India. Male albino Wistar rats (200-250 g) were used and the animals were divided into five groups of six animals each. The control-group I, standard-group II, test-group III, test-group IV, and test-group V received cholesterol, pure drug suspension plus cholesterol, E-CD plus cholesterol, ECD-TPGS plus cholesterol, and E-CD-AA2G plus cholesterol, respectively. The animals were housed in polypropylene cages and kept at standard laboratory conditions $\left(25^{\circ} \mathrm{C} \pm 2^{\circ} \mathrm{C}\right.$ and $55 \% \pm 5 \% \mathrm{RH})$. Six animals per cage were accommodated with free access to standard laboratory diet (Lipton feed, Mumbai, India) and water ad libitum.

The antihypercholesterolemic study was carried out for 28 days, and the animals were fed and dosed orally using 18gauge oral feeding needle. To carry out the study, all the groups were induced with hypercholesterolemia by administering them with high-fat diet $(200 \mathrm{mg}$ cholesterol in $2 \mathrm{~mL}$ coconut oil). Two hours following the administration of high- 
fat diet, the treatment groups, II, III, IV, and V, were, respectively, fed with pure drug, E-CD, E-CD-TPGS, and E-CDAA2G, dispersed in $0.25 \% w / v$ NaCMC. The daily dose for rats was calculated by considering the rat-to-human being surface area ratio [2, 27]. Blood samples were collected on day $7,14,21$, and 28 , after anesthetizing the rats with diethyl ether, by retro-orbital puncture, into anticoagulated microcentrifuge tubes (heparin treated). The plasma was separated by centrifugation at $5000 \mathrm{rpm}$ for $20 \mathrm{~min}$ and stored at $2{ }^{\circ} \mathrm{C}$ until further use. Percent reduction in the levels of total cholesterol (TC) was analyzed using and in vitro Cogent diagnostic kit (Span Diagnostics Ltd., Surat, India).

\section{Statistical Analysis}

All the results were shown as mean \pm SD. The data pertaining to solubility, $\log P$, and dissolution investigations were analyzed by one-way analysis of variance followed by post hoc Tukey multiple comparison test ( $p$ value set 0.05 ). The antihypercholesterolemic study results were analyzed by two-way analysis of variance followed by post hoc Bonferroni multiple comparison test.

\section{RESULTS}

\section{Evaluating the Effect of Increasing Concentration of TPGS or AA2G with Fixed Concentration of HPBCD}

The solubility of Eze, in a fixed $2 \% w / v$ HPBCD solution ( $\mathrm{pH} 4.5)$ in the presence of increasing concentrations of TPGS, increased until an optimum level and declined beyond that level (Fig. 2a). The best and minimum concentration of TPGS that could be used was selected as $0.05 \% \mathrm{w} / \nu$. The solubility of Eze, in a fixed $2 \% w / v$ HPBCD solution $(\mathrm{pH}$ $4.5)$ in the presence of increasing concentrations of $\mathrm{AA} 2 \mathrm{G}$, increased with the increase in AA2G concentration (Fig. 2b). The best and minimum concentration of AA2G was selected as $0.1 \% w / v$.

\section{Phase Solubility Studies}

The PS diagram of Eze in $4.5 \mathrm{pH}$ acetate buffer in the presence of HPBCD, alone and in combination with TPGS/AA2G, is presented in Fig. 2c. According to Higuchi and Connors [20], the PS curves of the liquid-state E-CD, ECD-TPGS, and E-CD-AA2G systems were classified as $A_{P}$, $A_{P}$, and $A_{L}$ types, respectively; the slopes of the straight line portions of the PS curves were 0.0091, 0.01, and 0.0169, respectively. The results indicated the occurrence of EzeHPBCD complex in the ratios 1:2, 1:2, and 1:1, in the systems E-CD, E-CD-TPGS, and E-CD-AA2G, respectively. The ratios were further confirmed by constructing a Job's plot (Fig. 2d). Benesi-Hildebrand plots are provided in Fig. 3.

The shift in the PS curve of liquid-state E-CD-AA2G to $A_{L}$ as opposed to $A_{P}$ nature of $E-C D$ binary system indicated the plausibility of formation of a water-soluble 1:1 EzeHPBCD complex in the presence of AA2G. The possibility that the addition of a water-soluble auxiliary substance can cause a shift in the PS curve has already been suggested in literature $[22,28]$. The stability constants were calculated from the straight line portions of the PS curves. The stability constant values of the liquid-state E-CD, E-CD-TPGS, and E-CD-AA2G systems were 1836.7 $\pm 9.2 \mathrm{M}^{-1}, 2020.2$ $\pm 10.1 \mathrm{M}^{-1}$, and $3438.1 \pm 9.9 \mathrm{M}^{-1}$, respectively.

\section{Characterization}

The FTIR spectra of all the samples are shown in Fig. 4a, and the spectral band assignments of the parent compounds are listed in Table I. Most of the principal absorption bands of pure Eze disappeared in the FTIR spectra of binary and ternary inclusion complexes, but none of the inclusion complexes exhibited new peaks dismissing the possibility of formation of chemical bonds during the $\mathrm{CD}$ complexation. The spectra of the binary and ternary inclusion complexes were similar to those of HPBCD. The one-dimensional H-NMR chemical shifts were reported as parts per million. The chemical shift values of the various protons of Eze (Fig. 1) are given in Table II. As shown in Fig. 4c, the DSC curve of Eze was characterized by a very sharp peak at $163.56^{\circ} \mathrm{C}$ with an onset at $163.07^{\circ} \mathrm{C}$ and end set at $165.74^{\circ} \mathrm{C}$. The broad endotherm of $\mathrm{HPBCD}$ between $50^{\circ} \mathrm{C}$ and $110^{\circ} \mathrm{C}$ suggested loss of water molecules from the CD cavity [29]. AA2G and TPGS displayed peaks at $170.72^{\circ} \mathrm{C}$ and $40.43^{\circ} \mathrm{C}$, respectively. The thermograms of all the formulations were similar to HPBCD alone. All the formulations presented the characteristic broad endotherm of HPBCD and the peaks corresponding to pure drug and ternary substances totally disappeared. Figure 4b shows X-ray diffractograms of all the samples. The graph of drug showed characteristic sharp diffraction peaks confirming the crystalline nature. Major intense peaks of drug were recorded at $2 \theta$ values of $7.825,13.859,15.733,17.136,18.589$, $19.345,19.845,21.719,22.866,23.363,25.21,26.96,28.16$, 30.045, and 32.96. The XRD pattern of AA2G and TPGS showed peaks characteristic of their respective crystal habit whereas that of HPBCD was diffused and scattered depicting its amorphous nature. The absence of characteristic drug peaks in the profiles of the binary and ternary complexes suggested loss of drug crystallinity due to complete entrapment into the CD cavity. The XRD profiles of the binary and ternary systems assumed the amorphous halo pattern typical of HPBCD indicating formation of new complexes and complete amorphization of the drug. The SEM images of all the samples are shown in Fig. 5. Pure Eze existed as small stoneshaped crystals whereas HPBCD appeared as irregularly large and small compact block solids with a thick and non-smooth surface. The microphotographs of TPGS could not be drawn on account of its waxy consistency. AA2G appeared as crystalline cylindrical particles. The stone-shaped drug appearance was completely disguised in the binary and ternary systems. E-CD complex appeared as glossy amorphous aggregates with smooth surface indicating the disappearance of the original morphology of drug and HPBCD and confirmed the formation of an intrinsic inclusion complex. E-CD-TPGS system looked like processed rectangular blocks with a slightly rough-surfaced appearance which might be due to the adsorption offered by TPGS as waxy layering in the ternary complex. The thick surface adherence of AA2G was quite prominent in the microphotographs of E-CD-AA2G ternary system. AA2G, upon lyophilization, might have assumed a more polished and radiant look, and the same was reflected in the SEM pictures of E-CD-AA2G. 

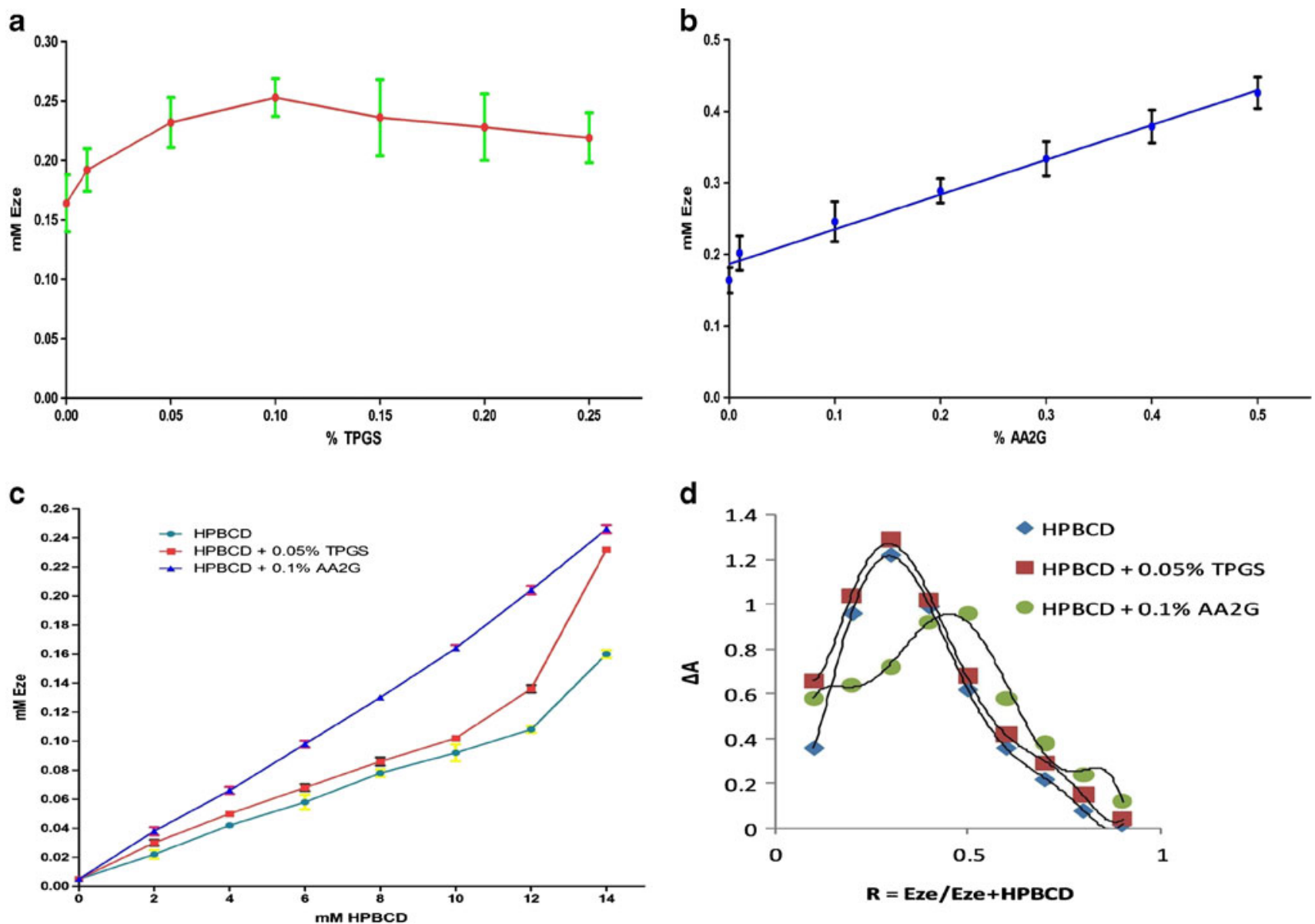

Fig. 2. Eze solubility diagram in a fixed concentration of $\operatorname{HPBCD}(2 \% w / v)$ with a increasing concentrations of TPGS, b increasing concentrations of AA2G, and $\mathbf{c}$ phase solubility curves of liquid-state binary and ternary systems. d Job's plots of liquid-state binary and ternary systems; studies were carried out using acetate buffer solutions of $\mathrm{pH} 4.5$

\section{Drug Content, Octanol-Water Partition Coefficient $(P)$ and Log $P$, Saturation Solubility Studies, Dissolution, and Antihypercholesterolemic Activity}

The drug content analysis was performed in triplicate and the average was reported. The percentages of drug content in E-CD, E-CD-TPGS, and E-CD-AA2G were found to be $98.9 \% \pm 1.89 \% w / w, 100.21 \% \pm 1.02 \% w / w$, and $99.99 \% \pm 1.32 \% w / w$, respectively. The results indicated that the drug was uniformly distributed in all the complexes. The experimental results of $\log P$ measurements are tabulated in Table III. $\Delta \log P$ (the relative hydrophilicity enhancement) was also determined to express the improved hydrophilicity brought about by HPBCD complexation and addition of auxiliary substances to the binary complex. The $\Delta \log P$ can be defined as $\Delta \log P=\log P$ guest (pure drug) $-\log P$ complex (binary/ternary) [30]. The aqueous saturation solubility results of pure drug and binary and ternary systems are shown in Table III. The dissolution efficiency (DE), $t_{80 \%}$, and $t_{90 \%}$ values are calculated for each of the systems and tabulated in Table IV. The dissolution profiles of all the systems are shown in Fig. 6. The percent reduction in the levels of TC achieved by various treatment groups is presented in Fig. 7 .

\section{DISCUSSION}

The primary objective of the current study was to increase HPBCD solubilization of Eze by introducing TPGS/ AA2G as ternary components. The liquid-state basic solubility analysis ("Evaluating the Effect of Increasing Concentration of TPGS or AA2G with Fixed Concentration of HPBCD") confirmed the increase in solubility pattern of Eze with the increase in the amounts of TPGS/AA2G in the presence of a fixed concentration of HPBCD suggesting the existence of possible intermolecular interactions among Eze, HPBCD, and TPGS/AA2G. The study also suggested the concentration of auxiliary component to be used to obtain a stable as well as more soluble ternary system. Both TPGS and AA2G caused statistically significant improvement in the stability constant compared to binary Eze-CD $(p<0.05)$ indicating the formation of more stable ternary complexes.

With respect to the binary E-CD system, the altered $\mathrm{pH}$ could be the possible reason for the higher stability constant obtained in this study (statistically significant, $p<0.05$ ) compared to the value reported earlier $\left(1316 \mathrm{M}^{-1}\right)$ by Patel et al. [5]. We performed all the liquid-state analyses using $4.5 \mathrm{pH}$ acetate buffer in contrast to the distilled water used by Patel et al. [5] because our dissolution studies were conducted using 

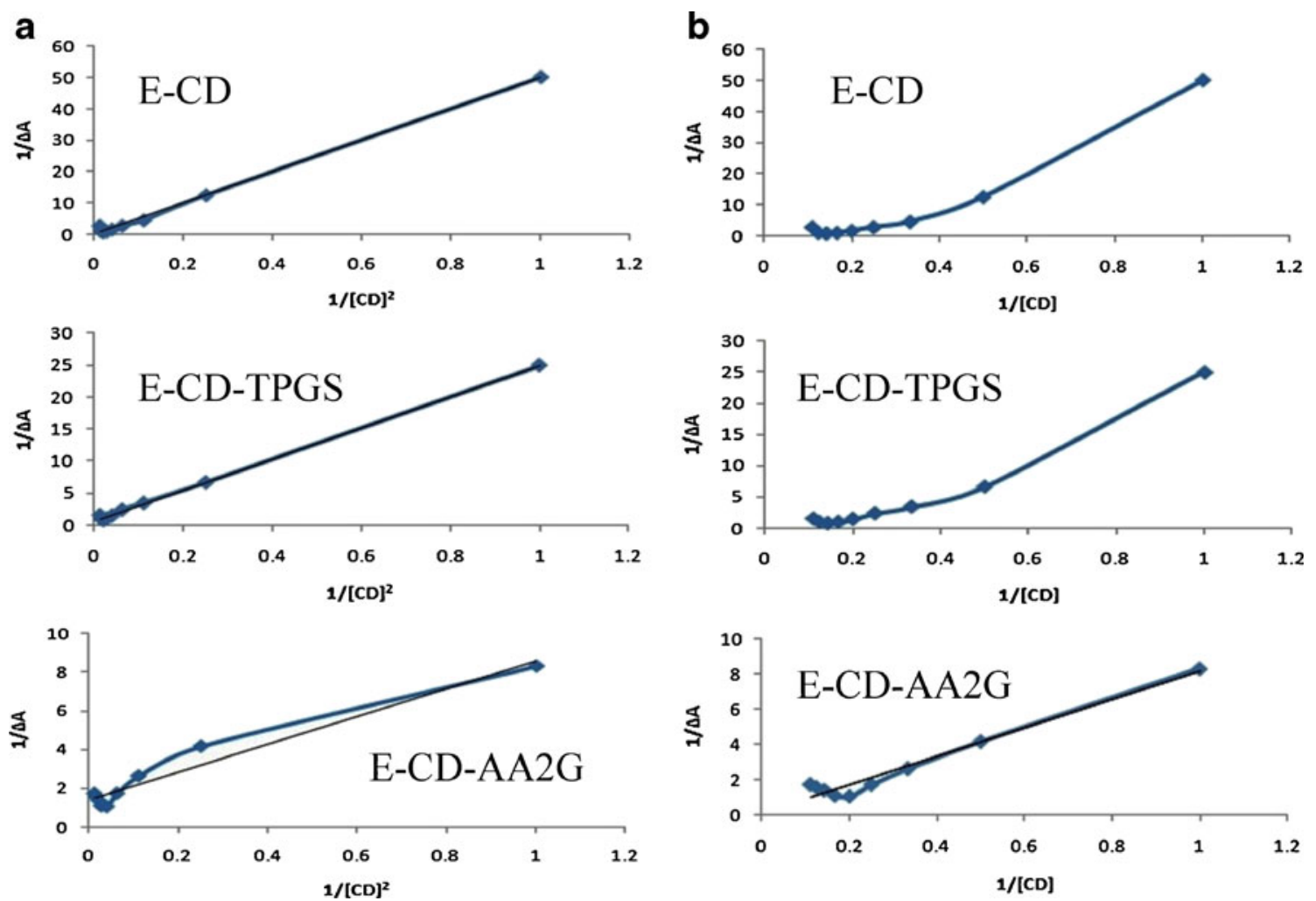

Fig. 3. Benesi-Hildebrand plots. a Double reciprocal plots of binary and ternary systems and $\mathbf{b}$ reciprocal plots of binary and ternary systems

the same $\mathrm{pH}$ media. The $\mathrm{pH}$ of the FDA-recommended dissolution media for Eze is also the same [26]. Both TPGS and AA2G further enhanced the solubility and stability of E-CD. The Eze-HPBCD complex occurred in the ratios 1:2, 1:2, and $1: 1$ in the systems E-CD, E-CD-TPGS, and E-CD-AA2G, respectively. The PS results and Job's and Benesi-Hildebrand plots confirmed that the addition of AA2G successfully decreased the amount of HPBCD required to complex and completely solubilize Eze.

Solid inclusion complexes were prepared as described in "Preparation of Inclusion Complexes in Solid State." The solidstate characterization suggested that all the graphs of binary and a

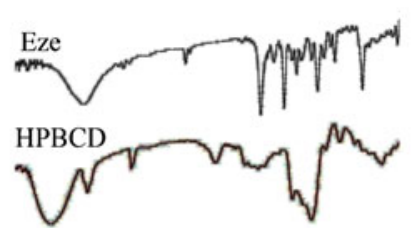

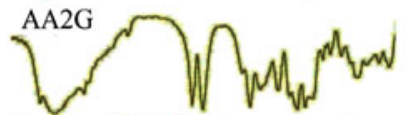
$\therefore \sqrt{\mathrm{TPGS}^{m}} \|_{\text {Mhr }}^{m} N^{M}$ E-CD $\sqrt{7}$

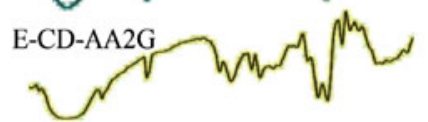
E-CD-TPGS

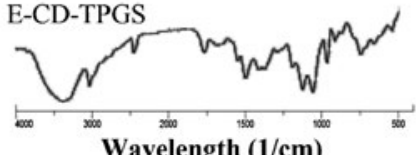

b
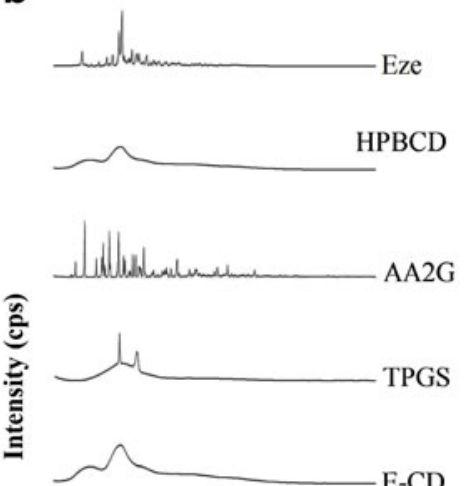

E-CD-AA2G

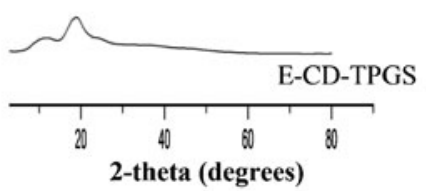

C
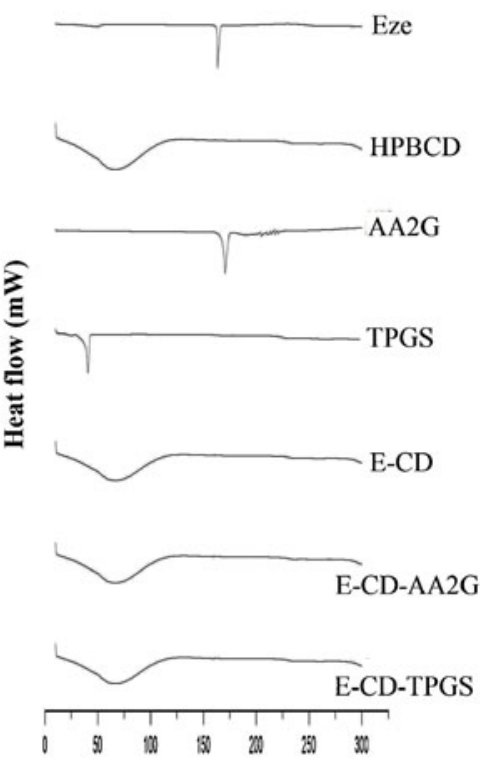

Temperature (degrees centigrade)

Fig. 4. a FTIR spectra, $\mathbf{b}$ X-ray diffractograms, and $\mathbf{c}$ DSC thermograms 
Table I. FTIR Data Table Presenting Characteristic Peak Assignments of Parent Compounds

P a r e n t compound FTIR spectral bands and assignments

Eze

(2)

$3267.52 \mathrm{~cm}^{-1}$ (broad stretching of intermolecular hydrogen bonded $\mathrm{O}-\mathrm{H}$ ); $2914.54 \mathrm{~cm}^{-1}$ (stretching of aromatic C-H); $2958.9 \mathrm{~cm}^{-1}$ (stretching of aliphatic C-H); $1886.44 \mathrm{~cm}^{-1}$ (overtone band of lactone ring); $1718.69 \mathrm{~cm}^{-1}$ (stretching of $\mathrm{C}=\mathrm{O}$ of lactone ring); $1614.47 \mathrm{~cm}^{-1}$ (vibration band of ring skeleton); $1510.31 \mathrm{~cm}^{-1}$ (stretching of ring C-C), 1404.22 and $1444.73 \mathrm{~cm}^{-1}$ (stretching of $\mathrm{C}-\mathrm{N}$ ); $1354.07 \mathrm{~cm}^{-1}$ (bending of in plane $\mathrm{O}-\mathrm{H}$ ); 1273.06, 1220.98, and $1165.04 \mathrm{~cm}^{-1}$ (stretching of C-F); 1066.67 and $1107.18 \mathrm{~cm}^{-1}$ (stretching of C-O of secondary alcohol); $1016.52 \mathrm{~cm}^{-1}$ (ring stretching of cyclobutanes); $941.29 \mathrm{~cm}^{-1}$ (ring vibration of alkyl cyclobutanes); and $825.56 \mathrm{~cm}^{-1}$ (ring vibration of para-disubstituted benzene)

HPBCD Intense bands between 3300 and $3500 \mathrm{~cm}^{-1}$ (O-H stretching vibrations) and between 2800 and $3000 \mathrm{~cm}^{-1}$ region $(-\mathrm{CH}$ and $\mathrm{CH}_{2}$ group vibrations)

AA2G $3292.60 \mathrm{~cm}^{-1}$ (O-H stretch), 1770.71 and $1705.13 \mathrm{~cm}^{-1}$ (C=O stretch), and $1400.27 \mathrm{~cm}^{-1}$ (O-H bending)

TPGS ternary systems were similar to the graphs of HPBCD because with the increase in complexation efficiency and addition of TPGS/AA2G, an excess of free HPBCD may have been present in each of the inclusion complex samples [30].

FTIR and NMR spectra were drawn to confirm the formation of inclusion complexes. It has been described in some past publications that the formation of ternary complexes is hard to be determined by either of the spectroscopic techniques due to the overlapping spectral readings [6, 31, 32]. Only the existence of the Eze-CD complex in all the systems may be inferred. In all binary and ternary systems, no new FTIR peaks different from those of parent compounds were noted, but modifications like broadening, attenuation, and frequency shifts in the characteristic bands of the drug were observed. The hydrophobic regions of Eze are likely to get placed into the lipophilic core of HPBCD. The inclusion complexation could have involved formation of hydrogen bonds between the drug and HPBCD which was also suggested by the proton shifts recorded in H-NMR studies. The NMR spectrum of free drug was the same as reported by Guntupalli et al. [33]. Eze has 13 different types of protons and some protons experienced upfield shifts and others, downfield. Such variations might have occurred possibly due to the steric effects from HPBCD, slight variations in local polarity, and differential shielding undergone due to van der Waals interactions with HPBCD and TPGS/AA2G. The inclusion could have become stabilized by the formation of hydrogen bonding (a) between the electronegative atoms of the Eze molecule and the protons of the HPBCD molecule (the interior $\mathrm{H} 3$ proton located at the wide side of the $\mathrm{CD}$ cavity and the $\mathrm{H} 5$ proton located at the narrow side of the cavity) [34] and (b) between the oxygen atom of HPBCD and the hydrophobic alkyl or aryl protons of Eze or may be even the alkyl hydroxyl protons of Eze. The ppm shifts quantitatively demonstrated the stability of inclusion complexes and the depth of ligand penetration into $C D$ cavity. Based on the chemical shift values, it may be inferred that Eze molecule could have been captivated by HPBCD cavity in all the binary and ternary systems. An intrinsic inclusion complex formation may be inferred though the exact orientation of Eze in the CD cavity needs to be established.

The DSC and XRD graphs confirmed complete amorphization of binary and ternary systems similar to Selic et al. [7] and unlike the incompletely amorphous Eze-HPBCD complexes reported in the earlier works $[5,6]$. The auxiliary components could have synergistically favored the drug entrapment into CD cavity. The complete amorphization confirmed complete drug complexation with the use of TBA to solubilize Eze solution and highlighted the efficient complexing power of HPBCD toward the drug through molecular interactions among drug, HPBCD, and TPGS/AA2G.

The SEM photomicrographs finally confirmed the formation of new binary and ternary complexes of Eze with hydrophilic HPBCD and ternary components in the solid state as all the processed samples exhibited unique morphological properties. All the three systems, E-CD, E-CD-TPGS, and E-

Table II. NMR Data Table Presenting Protonic Shifts of Eze Protons After Binary and Ternary Complexation (Chemical Shifts Values in ppm)

\begin{tabular}{|c|c|c|c|c|c|c|c|}
\hline Proton & Free Eze & $\mathrm{E}-\mathrm{CD}$ & $\Delta \mathrm{E}-\mathrm{CD}$ & $\mathrm{E}-\mathrm{CD}-\mathrm{AA} 2 \mathrm{G}$ & $\Delta \mathrm{E}-\mathrm{CD}-\mathrm{AA} 2 \mathrm{G}$ & E-CD-TPGS & $\Delta \mathrm{E}-\mathrm{CD}-\mathrm{TPGS}$ \\
\hline $1,1^{1}$ & 7.110 & 7.135 & 0.025 & 7.125 & 0.015 & 7.134 & 0.024 \\
\hline $2,2^{1}$ & 7.187 & 7.206 & 0.019 & 7.206 & 0.019 & 7.218 & 0.031 \\
\hline 3 & 5.275 & 5.866 & 0.591 & 5.706 & 0.431 & 5.764 & 0.489 \\
\hline 4 & 4.48 & 4.4 & 0.084 & 4.391 & 0.093 & 4.394 & 0.09 \\
\hline 5 & 1.708 & 1.703 & 0.005 & 1.701 & 0.007 & 1.715 & 0.007 \\
\hline 6 & 1.708 & 1.703 & 0.005 & 1.701 & 0.007 & 1.715 & 0.007 \\
\hline 7 & 3.337 & 3.391 & 0.054 & 3.372 & 0.035 & 3.389 & 0.052 \\
\hline 8 & 4.792 & 4.782 & 0.01 & 4.786 & 0.006 & 4.793 & 0.001 \\
\hline $9,9^{1}$ & 7.187 & 7.206 & 0.019 & 7.206 & 0.019 & 7.218 & 0.031 \\
\hline $10,10^{1}$ & 6.725 & 6.747 & 0.022 & 6.747 & 0.022 & 6.758 & 0.033 \\
\hline 11 & 9.511 & 9.505 & 0.006 & 9.505 & 0.006 & 9.498 & 0.013 \\
\hline $12,12^{1}$ & 7.263 & 7.283 & 0.02 & 7.281 & 0.018 & 7.287 & 0.024 \\
\hline $13,13^{1}$ & 7.110 & 7.135 & 0.025 & 7.125 & 0.015 & 7.134 & 0.024 \\
\hline
\end{tabular}



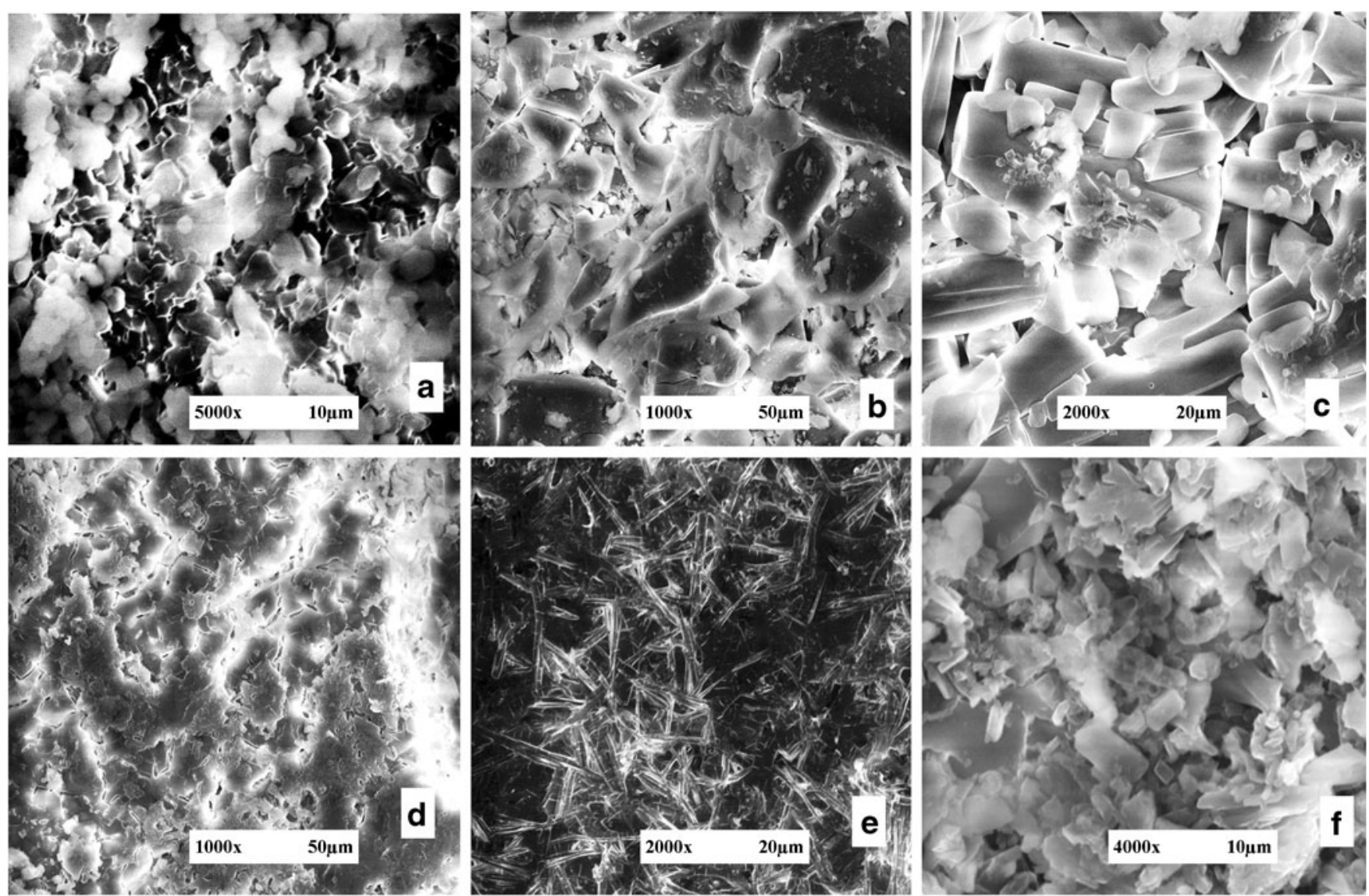

Fig. 5. SEM photomicrographs of a Eze, b HPBCD, c AA2G, d E-CD, e E-CD-AA2G, and f E-CD-TPGS

CD-AA2G, appeared not only different from the parent compounds but also different from each other. The altered particle shape, surface characteristics, and intimate bonding with the hydrophilic excipients in complexes may have contributed to enhanced solubility and dissolution rate of Eze [35]. Beyond the results from solid-state analysis, it is the remarkable enhancement in solubility and release properties that could be seen as proof for the ternary complex formation and for possible interactions between HPBCD/Eze and TPGS/AA2G.

The octanol-water partition coefficient, $P$, is the measure of the differential solubility of drug in octanol and water, and $\log P$ is Hansch factor and indicates the lipophilicity [21, 30]. The $\log P$ results were in the order Eze $>$ E-CD $>$ E-CDTPGS $>$ E-CD-AA2G ( $p<0.05$ for each comparison). Eze depicted low hydrophilicity, and this property was significantly improved by complexation with HPBCD and with the use of ternary components. The results suggested improvement in the hydrophilicity of drug by 2-2.5 times by binary and ternary complexation, and E-CD-AA2G system showed the highest hydrophilicity. The solubility of Eze in distilled water improved significantly up to 6-7.7-fold by binary and ternary complexation in comparison to pure drug $(p<0.05$ for each complex in comparison to pure Eze). CD complexation caused amorphization of drug and significantly improved the solubility of the drug by decreasing the surface tension between the medium and drug. Introduction of TPGS or AA2G successfully increased HPBCD solubilization of Eze. E-CDTPGS and E-CD-AA2G systems might be novel, but there are no reported results for the $\log P$ and aqueous solubility of binary E-CD system too, in the literature. The solubility of each of the ternary complex was significantly higher than that of E-CD $(p<0.05)$, but the difference in the solubilities of ECD-AA2G and E-CD-TPGS was not statistically significant $(p>0.05)$. However, it needs to be noted that the amount of HPBCD employed in E-CD-AA2G was half that of E-CDTPGS system.

TPGS is an amphiphilic molecule which means it contains both hydrophilic and lipophilic groups. The lipophilic portion of the molecule may tend to get attracted toward the CD cavity and may pose competition to the drug. However, CDguest complexes are formed at definitive ratios and the ratio is characteristic of the guest molecule. In liquid-state solubility study, "Evaluating the Effect of Increasing Concentration of TPGS or AA2G with Fixed Concentration of HPBCD," a decrease in the solubility of drug was observed with increasing concentration of TPGS beyond $0.1 \%$. When used above $0.1 \%$, TPGS might have competed with the drug for CD cavity and the free TPGS and CD molecules available for drug molecules could have been insufficient to solubilize the drug efficiently and eventually caused decrease in drug solubility. Such phenomenon of competition in the presence of surfactant excipients has already been reported [12]. Even if TPGS had not competed for CD cavity, there could have been some interaction between TPGS and the external surface of HPBCD which would have interfered with the drug affinity to the inner cavity of $\mathrm{CD}$. In case there was no prevalence of competition or interference posed by TPGS, the presence of two solubilizers could have contributed to a significant additive effect on the 
Table III. Saturation Solubility and $\log P$ Values (Data Shown as Mean $\pm \mathrm{SD}$ )

\begin{tabular}{lccr}
\hline System & Saturation solubility $\left(10^{-3} \mathrm{mg} / \mathrm{mL}\right)$ & $\log P$ & $\Delta \log P$ \\
\hline Eze & $1.99 \pm 0.62$ & $1.602 \pm 0.029$ & - \\
E-CD & $12.01 \pm 0.23$ & $0.765 \pm 0.061$ & $0.837 \pm 0.032$ \\
E-CD-TPGS & $14.55 \pm 0.18$ & $0.686 \pm 0.032$ & $0.916 \pm 0.003$ \\
E-CD-AA2G & $15.26 \pm 0.74$ & $0.646 \pm 0.054$ & $0.956 \pm 0.025$ \\
\hline
\end{tabular}

drug solubilization which did not happen. However, with the concentration of TPGS kept optimum in the solid ternary system $(0.05 \%)$, we assumed that the competition phenomenon subsided. Both CD and TPGS could have been available to the drug as solubilizers by inclusion complexation and by surfactant (micellar solubilization) effect, respectively [12]. While HPBCD is also known to cause non-inclusion type of micellization/self-association mode of solubilization of drugs, the surfactant activity of TPGS is likely to prevent the former possibility. Surfactant excipients, when used as ternary components, are known to prevent the formation of HPBCD aggregates [12]. TPGS could have completely coated the ECD inclusion complex (indicated by SEM studies) by interacting with both the drug and CD by weak intermolecular bonds and formed a stable E-CD-TPGS ternary system. The optimization of the proportions of ingredients of the solid ternary complex could have brought about the aqueous solubility enhancement in case of E-CD-TPGS ternary system.

AA2G is a completely hydrophilic molecule that lacks any affinity to the lipophilic CD cavity. This glucosidic watersoluble substance could have had a definitive synergistic effect on the $\mathrm{CD}$ drug solubilization as indicated by the liquid statesolubility study, "Evaluating the Effect of Increasing Concentration of TPGS or AA2G with Fixed Concentration of HPBCD." AA2G may not have diffused into the CD cavity but there is a possibility of hydrogen bond formation or dipole-dipole interaction between $\mathrm{CD}$ and AA2G which explains the shifting of PS curve of this liquid-state ternary system to $A_{L}$ type as compared to the $A_{P}$ type exhibited by the liquid-state binary system. Another possibility is that AA2G could have formed weak hydrogen or van der Waals bonding with drug or might have also favored the HPBCD's non-inclusion type of micellization/self-association mode of solubilization of the drug. With the increasing AA2G concentration, the solubility of drug also increased and a minimum quantity $(0.1 \%)$ of AA2G was chosen for the preparation of solid ternary complex. The chemical structure of AA2G is enriched with several hydroxyl groups which offer higher possibility to hydrogen bond with drug as well as HPBCD. As such, AA2G could have totally coated the E-CD inclusion complex (indicated by SEM studies) by interacting with both the drug and CD by weak intermolecular bonds and formed a stable E-CD-AA2G ternary system. The proportions of the components of solid ternary system chosen to formulate the E-CD-AA2G system were sufficient to achieve greater aqueous solubility of drug even at half the $\mathrm{CD}$ concentration (compared to E-CD and E-CD-TPGS).

The drug dissolution rate was greater for both binary and ternary systems, and the pure drug obviously presented the lowest dissolution rate because of its low solubility. DE was improved by 3-3.5-fold on binary and ternary complexation as compared to pure Eze. The DE values were significantly higher in the order E-CD-AA2G $>$ E-CD-TPGS $>$ E-CD $>$ Eze ( $p<0.05$ for each comparison). Pure drug did not dissolve more than $36.2 \% \pm 4.2 \%$ during the 120 -min dissolution study. Both $t_{80 \%}$ and $t_{90 \%}$ values were significantly lower in the order ECD-A A $2 \mathrm{G}<\mathrm{E}-\mathrm{CD}-\mathrm{TPGS}<\mathrm{E}-\mathrm{CD} \quad(p<0.05$ for each comparison).

The percent drug dissolved in $30 \mathrm{~min}$ for the systems ECD, E-CD-TPGS, and E-CD-AA2G was $58.1 \% \pm 3.3 \%$, $74.2 \% \pm 2.4 \%$, and $82.5 \% \pm 2.8 \%$, respectively. Patel et al. [5] conducted dissolution studies in phosphate buffer $(\mathrm{pH} 7.8$ and containing $1 \% \mathrm{w} / \mathrm{v}$ SLS) and reported $60.7 \%$ drug dissolution ( $p>0.05$ compared to our system) and Bandyopadhyay et al. [2] reported $67.04 \%$ drug dissolution $(p<0.05$ compared to our system) in $0.5 \% w / v$ SLS solution, within $30 \mathrm{~min}$, from their respective binary Eze-HPBCD systems. The differences may be attributed to the altered dissolution medium employed.

The antihypercholesterolemic performance of pure Eze was statistically insignificant in comparison to the control group ( $p>0.05$ ), and the performances of all the three formulations, E-CD , E-CD-TPGS, and E-CD-AA2G were significantly prominent when compared to either control $(p<0.001)$ or pure Eze $(p<0.001)$, on each of the test day. The improved performance of the three systems was also suggested by their enhanced in vitro solubility and dissolution profiles. While the difference in the percent reduction in total plasma cholesterol levels achieved by E-CD and E-CD-AA2G was statistically insignificant $(p>0.05)$, the performance of E-CD-TPGS in comparison to either E-CD or E-CD-AA2G was significantly higher as indicated in Table V. The E-CD and E-CD-AA2G systems had the contribution of HPBCD alone to the

Table IV. Dissolution Data Shown as Mean \pm SD $(N=6)$

\begin{tabular}{llrr}
\hline System & DE $(\%)$ & $t_{80 \%}(\mathrm{~min})$ & $t_{90 \%}(\mathrm{~min})$ \\
\hline Eze & $24.91 \pm 1.65$ & Not achieved & Not achieved \\
E-CD & $73.54 \pm 1.32$ & $57.14 \pm 1.2$ & $84.38 \pm 1.32$ \\
E-CD-TPGS & $82.08 \pm 1.44$ & $40.91 \pm 1.33$ & $56.25 \pm 1.28$ \\
E-CD-AA2G & $85.92 \pm 1.19$ & $29.27 \pm 1.36$ & $42.19 \pm 1.23$ \\
\hline
\end{tabular}




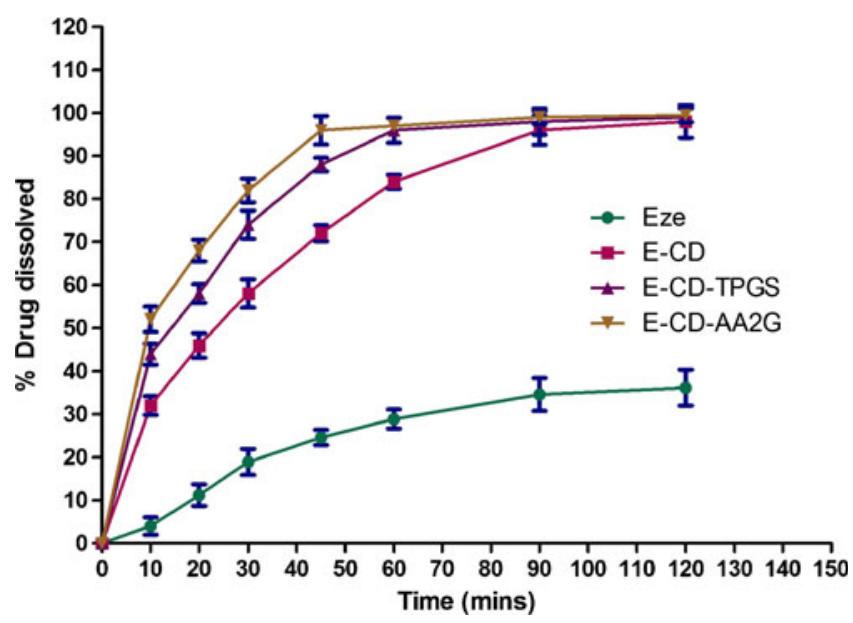

Fig. 6. Dissolution profiles of pure drug and binary and ternary complexes (vertical bars represent $\mathrm{SD}, N=6$ )

pharmacological activity (HPBCD maintains cholesterol homeostasis) [18]. The non-surfactant solubilizer, AA2G, played an insignificant role in improving the pharmacological action of Eze which suggests involvement of mechanisms other than solubility enhancement in explaining the superior hypocholesterolemic potential of E-CD-TPGS. The differentially superior performance of E-CD-TPGS in comparison to either E-CD or E-CD-AA2G may be explained by the agonistic contribution of HPBCD and P-gp inhibitory function of TPGS to the pharmacological action of Eze. Owing to the surfactant action, TPGS could have not only improved the solubility in vitro but have also altered the membrane permeability and inhibited the P-gp efflux of Eze at the intestine in vivo. Though $\mathrm{E}-\mathrm{CD}-\mathrm{AA} 2 \mathrm{G}$ presented superior in vitro dissolution profile, the markedly superior pharmacological performance of E-CD-TPGS may be ascribed to reduced oral bioavailability variations of Eze. Therefore, considering the statistically insignificant difference in the aqueous solubilities of E-CD-AA2G and E-CD-TPGS, the latter may be noted as the most effective formulation for oral delivery of Eze.

\section{CONCLUSION}

HPBCD solubilization of Eze was successfully improved by introducing TPGS/AA2G as ternary components in both solution and solid state. The surfactant properties of TPGS and the polyolic nature of AA2G served excellently in improving the drug aqueous solubility and dissolution properties. The use of solvent TBA enhanced the drug-CD complexation efficiency and caused complete amorphization of the drug in both binary and ternary systems. Both TPGS and AA2G favored the amorphous state and synergistically enhanced the surfactant action of HPBCD, increased the surface area and decreased the interfacial tension of drug particles on exposure to the dissolution medium, and prevented the aggregation of drug particles. AA2G best served as ternary component at in vitro level and decreased the amount of HPBCD required to solubilize Eze to half. However, considering the cumulative in vitro and in vivo performances, E-CD-TPGS may be noted as the best formulation to improve the solubility and oral absorption and reduce the bioavailability variations of Eze.

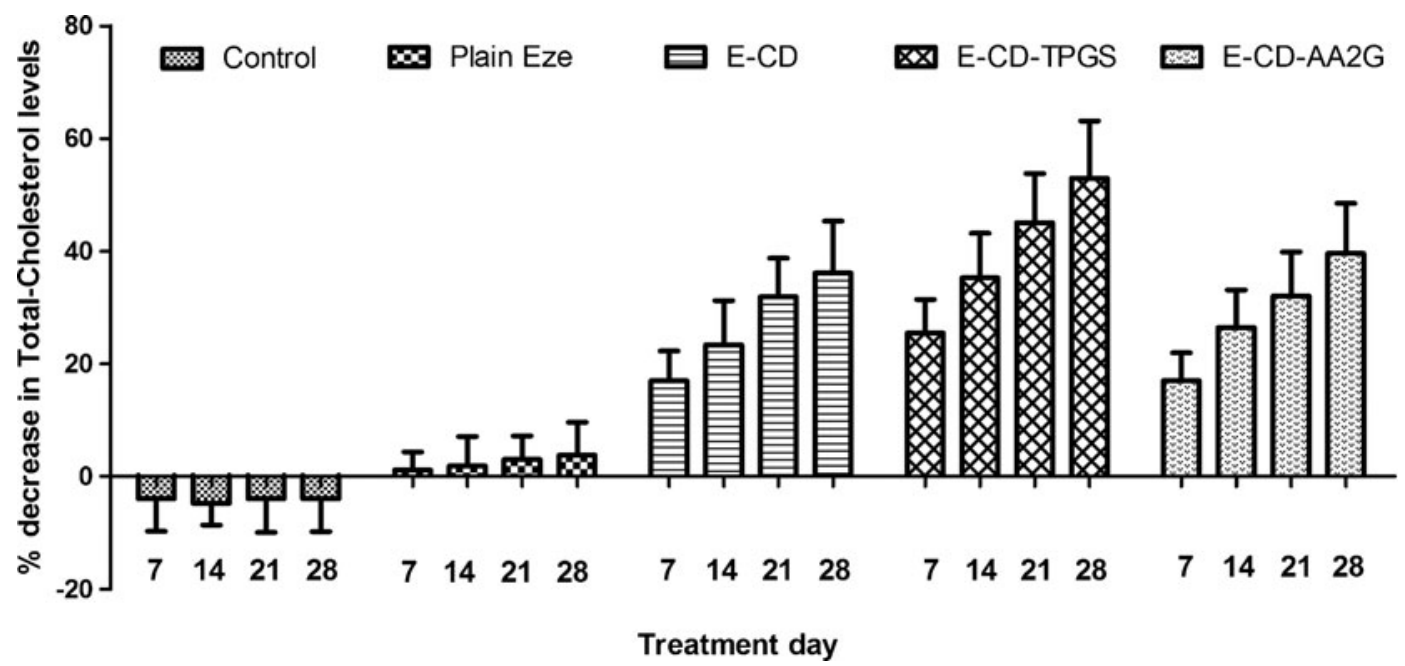

Fig. 7. Percent reduction in the total cholesterol levels achieved by various treatment groups (vertical bars represent $\mathrm{SD}, N=6)$ 
Table V. Statistical Significance of Difference in Antihypercholesterolemic Activity Between Selected Treatment Groups

\begin{tabular}{llll}
\hline Treatment groups under comparison & Day 7 & Day 14 & Day 21 \\
\hline E-CD and E-CD-TPGS & $p>0.05$ & $p<0.05$ & $p<0.01$ \\
E-CD-AA2G and E-CD-TPGS & $p>0.05$ & $p>0.05$ & $p<0.01 \quad p<0.001$ \\
\hline
\end{tabular}

\section{ACKNOWLEDGEMENTS}

The authors thank the anonymous reviewers for their valuable suggestions for our manuscript. The first author thanks Dr. ON Srivastava, Department of Physics, and the Head, Department of Chemistry, from BHU, Varanasi, and the Head, Department of Chemical Engineering, IIT (BHU), Varanasi, for providing XRD, SEM, NMR, and DSC facilities. The first author is thankful to the Director, IIT (BHU), Varanasi, India for providing institutional $\mathrm{PhD}$ fellowship in the form of teaching assistantship.

\section{REFERENCES}

1. Rosenblum SB, Huynh T, Afonso A, Davis HR, Yumibe N, Clader JW, et al. Discovery of 1-(4-fluorophenyl)-3(R)-[3-(4fluorophenyl)-3(S)-hydroxy-propyl]-4(S) (4-hydroxy phenyl)2-azetidinone ( $\mathrm{SCH} 58235)$ : a designed, potent, orally active inhibitor of cholesterol absorption. J Med Chem. 1998;41:973-80.

2. Bandyopadhyay S, Katare OP, Singh B. Optimized self nanoemulsifying systems of ezetimibe with enhanced bioavailability potential using long chain and medium chain triglycerides. Colloids Surf B: Biointerfaces. 2012;100:50-61.

3. Augustijns P, Brouwers J, Brewster ME. Supersaturating drug delivery systems: the answer to solubility-limited oral bioavailability? J Pharm Sci. 2009;98:2549-72.

4. Zoeller T, Dressman JB, Klein S. Application of a ternary HP- $\beta$ $\mathrm{CD}$-complex approach to improve the dissolution performance of a poorly soluble weak acid under biorelevant conditions. Int $\mathrm{J}$ Pharm. 2012;430:176-83.

5. Patel R, Bhimani D, Patel J, Patel D. Solid-state characterization and dissolution properties of ezetimibe-cyclodextrins inclusion complexes. J Incl Phenom Macrocycl Chem. 2008; $60: 241-51$

6. Taupitz T, Dressman JB, Klein S. New formulation approaches to improve solubility and drug release from fixed dose combinations: case examples pioglitazone/glimepiride and ezetimibe/simvastatin. Eur J Pharm Biopharm. 2013;84:208-18.

7. Selic L, Ham Z, inventors; Lek Pharmaceuticals, assignee, Inclusion complex of ezetimibe and a cyclodextrin and processes in the preparation thereof. European patent EP 1939174 A1. 2008 Jul 2.

8. Loftsson T, Frikdriksdóttir H, Sigurkdardóttir AM, Haruhisa U. The effect of water-soluble polymers on drug-cyclodextrin complexation. Int J Pharm. 1994;110:169-77.

9. Loh GOK, Tan YTF, Peh KK. Effect of HPMC concentration on $\beta$-cyclodextrin solubilization of norfloxacin. Carbohydr Polym. 2014;101:505-10.

10. Hirlekar RS, Sonawane SN, Kadam VJ. Studies on the effect of water-soluble polymers on drug-cyclodextrin complex solubility. AAPS PharmSciTech. 2009;10:858-63.

11. Chowdary KPR, Srinivas SJ. Influence of hydrophilic polymers on celecoxib complexation with hydroxypropyl $\beta$-cyclodextrin. AAPS PharmSciTech. 2006;7:E1-6. Article 79.

12. Nogueiras-Nieto L, Sobarzo-Sánchez E, Gómez-Amoza JL, Otero-Espinar FJ. Competitive displacement of drugs from cyclodextrin inclusion complex by polypseudorotaxane formation with poloxamer: implications in drug solubilization and delivery. Eur J Pharm Biopharm. 2012;80:585-95.
13. Sami F, Philip B, Pathak K. Effect of auxiliary substances on complexation efficiency and intrinsic dissolution rate of gemfibrozil- $\beta-C D$ complexes. AAPS PharmSciTech. 2010;11:27-35.

14. Jadhav P, Petkar B, Pore Y, Kulkarni A, Burade K. Physicochemical and molecular modeling studies of cefixime-l-arginine-cyclodextrin ternary inclusion compounds. Carbohydr Polym. 2013;98:1317-25.

15. Srivalli KMR, Lakshmi PK. Overview of P-glycoprotein inhibitors: a rational outlook. Braz J Pharm Sci. 2012;48:353-67.

16. Guo Y, Luo J, Tan S, Otieno BO, Zhang Z. The applications of Vitamin E TPGS in drug delivery. Eur J Pharm Sci. 2013;49:17586.

17. Inoue $\mathrm{Y}$, Yoshimura $\mathrm{S}$, Tozuka $\mathrm{Y}$, Moribe $\mathrm{K}$, Kumamoto $\mathrm{T}$, Ishikawa $\mathrm{T}$, et al. Application of ascorbic acid 2-glucoside as a solubilizing agent for clarithromycin: solubilization and nanoparticle formation. Int J Pharm. 2007;331:38-45.

18. Peake KB, Vance JE. Normalization of cholesterol homeostasis by 2-hydroxypropyl- $\beta$-cyclodextrin in neurons and glia from Niemann-Pick C1 (NPC1)-deficient mice. J Biol Chem. 2012;287:9290-8.

19. Soares-Sobrinho JL, Santos FLA, Lyra MAM, Alves LDS, Rolim LA, Lima AAN, et al. Benznidazole drug delivery by binary and multicomponent inclusion complexes using cyclodextrins and polymers. Carbohydr Polym. 2012;89:323-30.

20. Higuchi T, Connors KA. Phase solubility techniques. Adv Anal Chem Instrum. 1965;4:117-212.

21. Misiuk W, Zalewska M. Spectroscopic investigations on the inclusion interaction between hydroxypropyl- $\beta$-cyclodextrin and bupropion. J Mol Liq. 2011;159:220-5.

22. Jug M, Mennini N, Kövér KE, Mura P. Comparative analysis of binary and ternary cyclodextrin complexes with econazole nitrate in solution and in solid state. J Pharm Biomed Anal. 2014;91:8191.

23. Duran-Meras I, Pena AM, Salinas F, Caceres IR. Spectrofluorimetric determination of nalidixic acid based on host-guest complexation with $\gamma$-cyclodextrin. Analyst. 1994;119:1215-9.

24. Zeng J, Ren Y, Zhou C, Yu S, Chen W. Preparation and physicochemical characteristics of the complex of edaravone with hydroxypropyl- $\beta$-cyclodextrin. Carbohydr Polym. 2011;83:1101-5.

25. Wang D, Li H, Gu J, Guo T, Yang S, Guo Z, et al. Ternary system of dihydroartemisinin with hydroxypropyl- $\beta$-cyclodextrin and lecithin: simultaneous enhancement of drug solubility and stability in aqueous solutions. J Pharm Biomed Anal. 2013;83:141-8.

26. USFDA dissolution methods. http://www.accessdata.fda.gov/scripts/ cder/dissolution/dsp_SearchResults_Dissolutions.cfm?PrintAll=1. Last accessed 08 October 2014.

27. Dixit RP, Nagarsenker MS. Self-nanoemulsifying granules of ezetimibe: design, optimization and evaluation. Eur J Pharm Sci. 2008;35:183-92.

28. Jug M, Kosalec I, Maestrelli F, Mura P. Analysis of triclosan inclusion complexes with $\beta$-cyclodextrin and its watersoluble polymeric derivative. J Pharm Biomed Anal. 2011;54:1030-9.

29. Sinha VR, Anitha R, Ghosh S, Nanda A, Kumria R. Complexation of celecoxib with $\beta$-cyclodextrin characterization of the interaction in solution and in solid state. J Pharm Sci. 2005;94:676-87.

30. Misiuk W, Zalewska M. Investigation of inclusion complex of trazodone hydrochloride with hydroxypropyl- $\beta$-cyclodextrin. Carbohydr Polym. 2009;77:482-8.

31. Ammar HO, Salama HA, Mahmoud AA. Implication of inclusion complexation of glimepiride in cyclodextrin-polymer systems on its dissolution, stability and therapeutic efficacy. Int J Pharm. 2006;320:53-7. 
32. Valero M, Perez-Revuelta BI, Rodriguez LJ. Effect of PVPK-25 on the formation of the naproxen: beta-ciclodextrin complex. Int J Pharm. 2003;253:97-110.

33. Guntupalli S, Ray UK, Murali N, Gupta PB, Kumar VJ, Satheesh $\mathrm{D}$, et al. Identification, isolation and characterization of process related impurities in ezetimibe. J Pharm Biomed Anal. 2014;88:385-90.
34. Bernini A, Spiga O, Ciutti A, Scarselli M, Bottoni G, Mascagni P, et al. NMR studies of the inclusion complex between betacyclodextrin and paroxetine. Eur J Pharm Sci. 2004;22:445-50.

35. Yadav D, Yadav A, Karekar P, Pore Y, Gajare P. Enhanced solubility and dissolution rate of Olmesartan medoxomil using crystallo-co-agglomeration technique. Der Pharm Sin. 2012;3:160-9. 